\title{
Novel Hybrid Hydroxyapatite Spacers Ensure Sufficient Bone Bonding in Cervical Laminoplasty
}

\author{
Nobuhiro Tanaka ${ }^{1,2}$, Kazuyoshi Nakanishi ${ }^{2}$, Naosuke Kamei ${ }^{2}$, Toshio Nakamae ${ }^{2}$, \\ Shinji Kotaka ${ }^{2}$, Yoshinori Fujimoto ${ }^{1}$, Mitsuo Ochi ${ }^{2}$, Nobuo Adachi ${ }^{2}$ \\ ${ }^{1}$ Department of Orthopaedic Surgery, Hiroshima General Hospital, Hatsukaichi, Japan \\ ${ }^{2}$ Department of Orthopaedic Surgery, Institute School of Biomedical Sciences, Hiroshima University, Hiroshima, Japan
}

\section{Study Design: Prospective observational study.}

Purpose: This prospective analysis aimed to evaluate the efficacy and bone-bonding rate of hybrid hydroxyapatite (HA) spacers in expansive laminoplasty.

Overview of Literature: Various types of spacers or plates have been developed for expansive laminoplasty.

Methods: Expansive open-door laminoplasty was performed in 146 patients with cervical myelopathy; 450 hybrid HA spacers and 41 autogenous bone spacers harvested from the spinous processes were grafted into the opened side of each lamina. The patients were followed up using computed tomography (CT), and their bone-bonding rates for hybrid HA and autogenous spacers, bone-fusion rates of the hinges of the laminae, and complications associated with the implants were then examined.

Results: Clinical symptoms significantly improved in all patients, and no major complications related to the procedure were noted. The hybrid HA spacers exhibited sufficient bone bonding on postoperative CT. The hinges completely fused in over $95 \%$ patients within 1 year of the procedure. Only 4 spacers $(0.9 \%)$ developed lamina sinking, and most expanded laminae maintained their positions without sinking or floating throughout the follow-up period.

Conclusions: Hybrid HA spacers contributed to high bone-fusion rates of the spacers and hinges of the laminae, and no complications were associated with their use. Cervical laminoplasty with these spacers is safe and simple, and it yields sufficient fixation strength while ensuring sufficient bone bonding during the immediate postoperative period.

Keywords: Cervical vertebrae; Laminoplasty; Myelopathy compressive; Calcium hydroxyapatite

\section{Introduction}

Posterior spinal decompression via expansive laminoplasty was designed to preserve the posterior structures of the cervical spine and to prevent the postoperative development of instability and cervical kyphosis. The successful reconstruction of the laminae of vertebral arches is required for spinal cord decompression because the sink- ing or non-union of the expanded laminae may induce neurological regression, segmental motor paralysis, and postoperative axial pain. Various types of spacers or plates have been developed for expansive laminoplasty [1-6], and bone bonding after the rigid fixation of the expanded laminae may affect the clinical results following this procedure $[7,8]$. Interconnected porous calcium hydroxyapatite ceramic (IP-CHA) has been used as an artificial bone

Received Received Feb 7, 2018; Revised Apr 27, 2018; Accepted May 22, 2018

Corresponding author: Nobuhiro Tanaka

Department of Orthopaedic Surgery, Hiroshima General Hospital, Jigozen 1-3-3, Hatsukaichi, Hiroshima 738-8503, Japan

Tel: +81-829-36-3111, Fax: +81-829-36-5573, E-mail: nobutanaka65@hotmail.com 
substitute, and it provides superior biocompatibility and high osteoconductivity [9-13]. We developed novel hybrid hydroxyapatite (HA) spacers using IP-CHA and solid ceramic for expansive open-door laminoplasty. The purpose of the present prospective study was to evaluate the efficacy and bone-bonding rate of these hybrid HA spacers in cervical expansive laminoplasty.

\section{Materials and Methods}

IP-CHA, with its systematic arrangement of spherical uniform pores, was produced from a slurry of HA (Ca12 [PO4] 6[OH] 2) using the 'foam-gel technique' (NEOBONE; MMT Co. Ltd., Osaka, Japan). IP-CHA were sintered at $1,200^{\circ} \mathrm{C}$ with the following characteristics: porosity, 75\%; mean porous diameter, $150 \mu \mathrm{m}$; interconnective pore diameter, $10-90 \mu \mathrm{m}$ (mean, $40 \mu \mathrm{m}$ ), and pore interconnectedness, $>90 \%$. The compression strength of IP-CHA was 10-15 MPa because of the uniform arrangement of the pores.

The hybrid HA spacers used in this study were specifically designed for expansive laminoplasty (NEOBONE-X, NX05-001, 002, 003; MMT Co. Ltd.) (Fig. 1). The spacers are $9.3-10.3-\mathrm{mm}$ long, $10-\mathrm{mm}$ wide, and $4-\mathrm{mm}$ thick with an asymmetrical design; on the side of the opened laminae, the 'teeth' are on the outer side, whereas on the side of the facet joints, they are on the inner side. The center of the spacer comprises solid ceramic, while the peripheries comprise IP-CHA. Two small holes are present for suture fixation in the center.

A total of 146 (107 men; mean age, 66 years; age range,

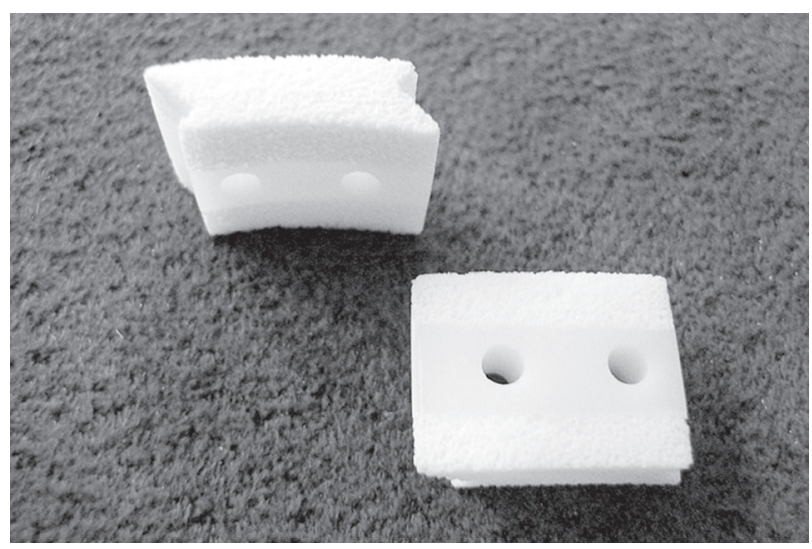

Fig. 1. Hybrid hydroxyapatite spacers specifically designed for expansive laminoplasty (model no., NX05-001, 002, 003; MMT Co. Ltd., Osaka, Japan).
30-92 years) consecutive patients with cervical myelopathy who underwent expansive open-door laminoplasty with hybrid HA spacers were prospectively examined in this study. All patients treated with cervical laminoplasty during the research period were enrolled in this study. Magnetic resonance imaging revealed either multiplelevel cord compression and/or spinal canal stenosis in all patients, making them eligible for cervical laminoplasty. Among the total cases, 108 cases were of cervical spondylotic myelopathy, 23 of ossification of the posterior longitudinal ligament, nine of cervical spondylotic amyotrophy, and six of intervertebral disc herniation. All patients showed signs or symptoms of cervical myelopathy and/ or radiculomyelopathy. The expansive open-door laminoplasty technique described by Hirabayashi et al. [14] was performed in all patients, and decompression was performed at the spinal levels C3-6 in 109 patients, C3-7 in 32 patients, $\mathrm{C} 3-5$ in four patients, and $\mathrm{C} 3-4$ in one patient. The hybrid HA spacers were preferentially transplanted between the opened side of the lamina and the lateral mass. If the resected spinous process, for instance, the $\mathrm{C} 6$ or $\mathrm{C} 7$ spinous process, was adequately long to accommodate autogenous bone spacers, a 10-12-mm long bone block was created and two small drill holes were made in each end of the graft for suture fixation. The autogenous bone spacers were alternately intermingled with the hybrid HA spacers on the opened side of each lamina, and they were firmly fixed with silicone-coated, braided nylon sutures. This study was reviewed and approved by the local institutional review board, and all patients provided informed consent prior to their inclusion in the study.

The patients were postoperatively followed up for at least 1 year for an average of 22 months (range, 12-86 months). During this time, all patients were examined using computed tomography (CT), whereby displacement, breakage, absorption, and bone fusion of grafted spacers could be evaluated at 3,6, and 12 months. Bone fusion with the spacer was defined as the disappearance of the gap at the interface between the grafted spacer and host bone, with new bone formation on both sides of the spacer, as visualized on CT. The bonding rate was measured as the percent of spacers for which this gap had disappeared. Cervical spine radiographs in the flexed and extended positions were captured to evaluate the range of motion (ROM) of the cervical spine preoperative and at 1 year postoperatively. The ROM was assessed by mea- 
suring the difference in C2-7 alignment between flexion and extension. The patient's neurological conditions were assessed using the scoring system developed by the Japanese Orthopaedic Association (JOA), and functional improvements in the JOA scores were determined from the recovery rate (RR) [14]. Axial pain in the neck was measured using a Visual Analog Scale (VAS; range, 0-10; with 0 denoting no pain). The bonding rates for both the hybrid HA and autogenous spacers, the bone-fusion rates of the hinges of the laminae, and complications associated with the implants were also examined. The unpaired Student $t$-test was used to perform statistical analyses for the comparison of the JOA scores and ROM, and chi-square test was performed to compare the bonding rates of the spacers. All $p<0.05$ were considered to be statistically significant.

\section{Results}

A total of 450 hybrid HA spacers and 41 autogenous spacers were grafted in 146 patients. The preoperative JOA scores averaged 11.5 points (range, $5-15$ points). The postoperative JOA scores at the time of the final follow-up (mean, 14.2 points; range, $8-17$ points) were significantly higher than the preoperative JOA scores $(p<0.05)$, and the mean RR was $52 \%$ (range, $8 \%-100 \%$ ). The operative time averaged 112 minutes (range, 60-220 minutes), and blood loss averaged $91 \mathrm{~mL}$ (range, 10-680 mL). No complications, such as allergic responses or inflammation, associated with the use of hybrid HA spacers were noted. The preoperative ROM of the cervical spine in the sagittal direction averaged $41^{\circ} \pm 13^{\circ}$ (range, $11^{\circ}-80^{\circ}$ ), and the postoperative ROM at 12 months averaged $33^{\circ} \pm 13^{\circ}$ (range, $\left.5^{\circ}-67^{\circ}\right)$. Although the postoperative ROM of the cervical spine was significantly lower $(p<0.05)$ than the preoperative value, the average ROM of the neck was preserved at $80 \%$ even after the surgery.

None of the hybrid HA spacers showed major breakages throughout the follow-up period. However, three spacers $(0.7 \%)$ showed minor breakage on CT at 3 months postoperatively (Fig. 2), and these spacers spontaneously fused with no further displacement, and the hinge of the expanded lamina was also fused on follow-up CT. Four spacers $(0.9 \%)$ in two patients developed lamina sinking subsequent to the displacement of the spacers, and one patient $(0.7 \%)$ required additional posterior fusion surgery with instrumentation because of the worsening of

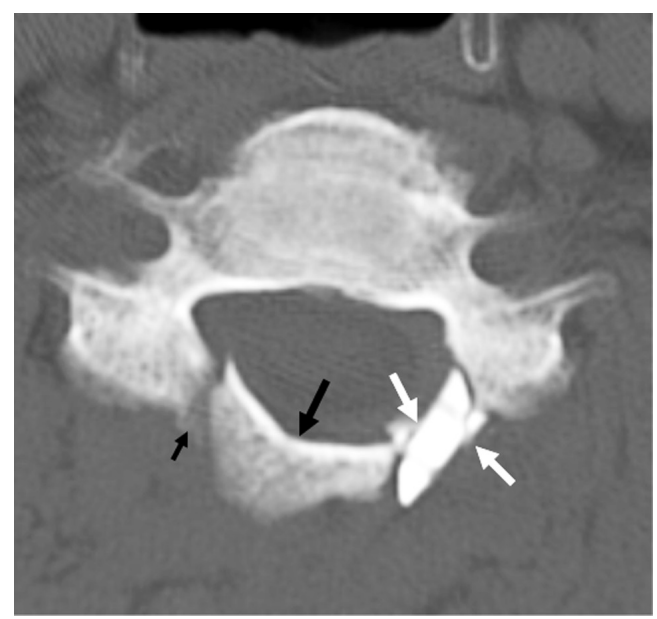

Fig. 2. A 3-month postoperative computed tomography revealing fine cracks (white arrow) in the hybrid hydroxyapatite spacer, expanded laminae (large black arrow), and the hinges of the laminae (small black arrow).

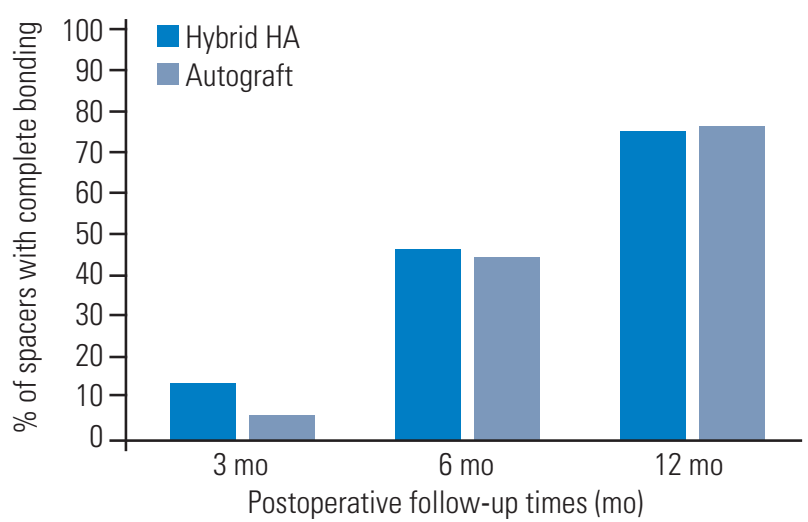

Fig. 3. The bone-bonding rates of hybrid $\mathrm{HA}$ and autogenous spacers. No significant differences were noted in the bonding rates between the different spacers $(p>0.05)$. HA, hydroxyapatite.

clinical symptoms associated with progressive kyphosis of the cervical spine accompanied by the sinking of the expanded laminae. One patient $(0.7 \%)$ complained of significant postoperative axial pain in the neck, reporting a score of $5 / 10$ on the VAS, albeit no spacer issues. Two patients (1.4\%) showed postoperative transient C5 palsy with no evidence of accompanying spacer problems.

The bone-bonding rates of the hybrid HA spacers were $14 \%$ at 3 months, $46 \%$ at 6 months, and $75 \%$ at 1 year postoperatively. The bonding rates of the autogenous spacers were 5,44 , and 77 , respectively, at the abovementioned time points (Fig. 3). Partial absorption was reported for $2 \%$ of the autogenous spacer at 3 months, $16 \%$ at 6 months, and $18 \%$ at 1 year postoperatively, whereas none of the hybrid HA spacers were absorbed. No signifi- 


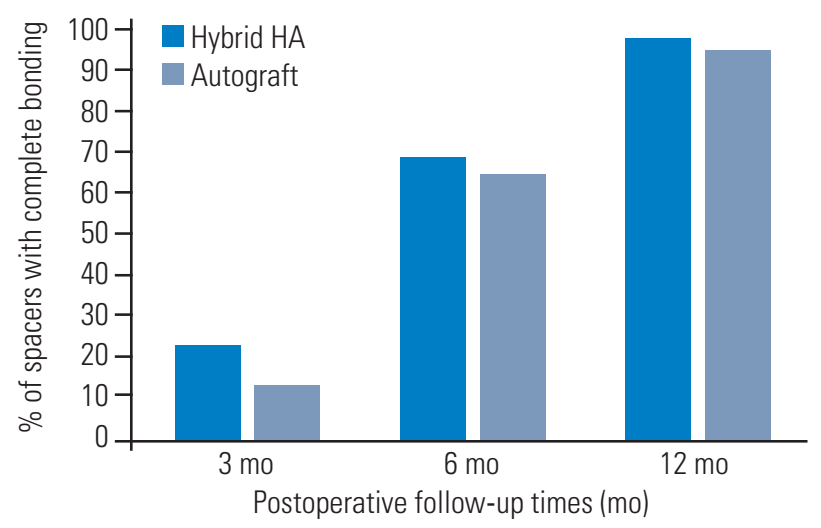

Fig. 4. The bone-bonding rates of the hinges of the expanded laminae with the hybrid $\mathrm{HA}$ and autogenous spacers. No significant differences were noted in the bonding rates of the hinges between the spacers ( $p>0.05)$. HA, hydroxyapatite.
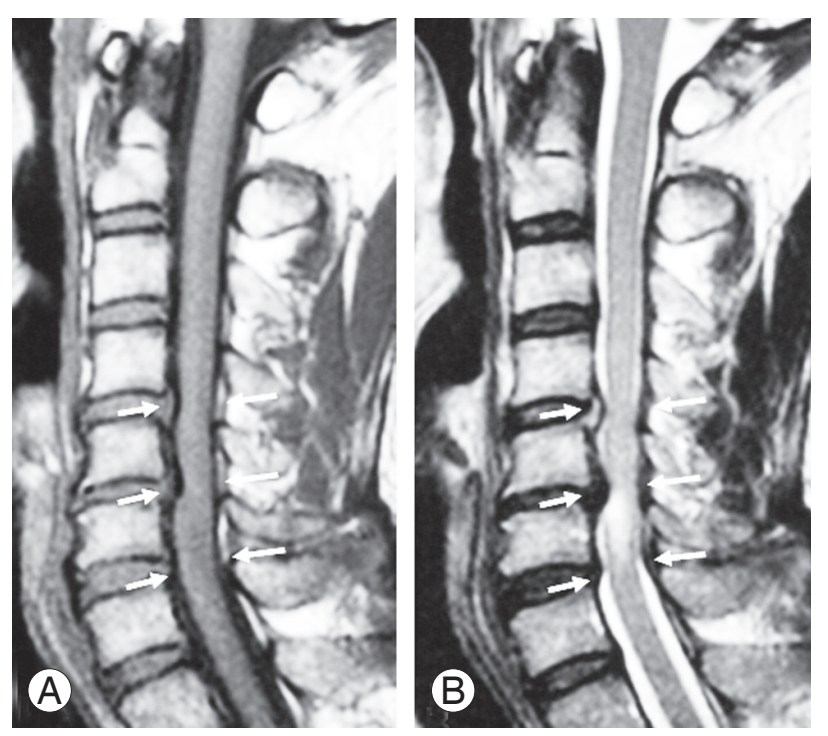

Fig. 5. Magnetic resonance imaging of a 44-year-old patient. Preoperative T1- (A) and T2-weighted (B) sagittal-view images revealing spinal canal stenosis at C4-5, C5-6, and C6-7 (arrows). cant differences were noted in the bonding rate between the spacer types. The hinges of the expanded laminae with the hybrid HA spacers were completely fused in $22 \%$ patients at 3 months, $69 \%$ patients at 6 months, and 97\% patients at 1 year and those with the autogenous spacers were fused in $15 \%, 65 \%$, and $95 \%$ of the patients at 3,6 , and 12 months, respectively (Fig. 4). No significant between-group differences were noted in the bonding rate of the hinges of the expanded laminae to the two different spacers.

\section{Case report}

A 44-year-old man presented with significant motor disturbance, numbness of both the hands, and spastic gait since 8 months. His magnetic resonance imaging revealed severe spinal canal stenosis at C4-5, C5-6, and C6-7 (Fig. 5). His preoperative JOA score was 11 points. Expansive laminoplasty was performed from $\mathrm{C} 3$ to $\mathrm{C} 7$, and hybrid HA spacers were grafted at C3, C4, C5, and C7. Autogenous spacers harvested from the spinous processes of $\mathrm{C} 7$ were grafted at C6. No complications related to either the surgery or the hybrid HA spacers occurred. Postoperative CT revealed good adaptation of the hybrid HA spacers without displacement or sinking, and those recorded at 6 months revealed bone bonding of the hybrid HA spacers and the hinge of the expanded laminae (Fig. 6). At 12 months postoperatively, the JOA score had improved to 14.5 points with an RR of $58 \%$, whereas the ROM of the cervical spine was $53^{\circ}$, with $90 \%$ of the preoperative ROM preserved (Fig. 7).
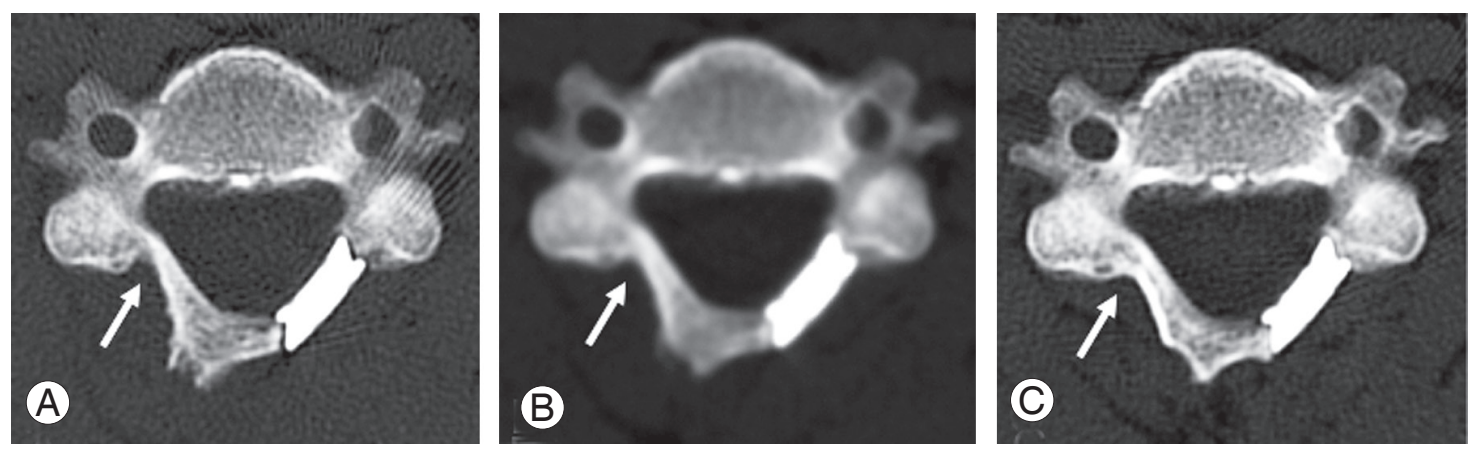

Fig. 6. Computed tomography at C5 recorded at 3 (A), 6 (B), and 12 (C) months postoperatively demonstrating good adaptation of the hybrid HA spacers without displacement or sinking. The hinge of the expanded laminae (arrows) fused at 6 months postoperatively and the hybrid HA spacer completely fused at 6 months postoperatively. HA, hydroxyapatite. 

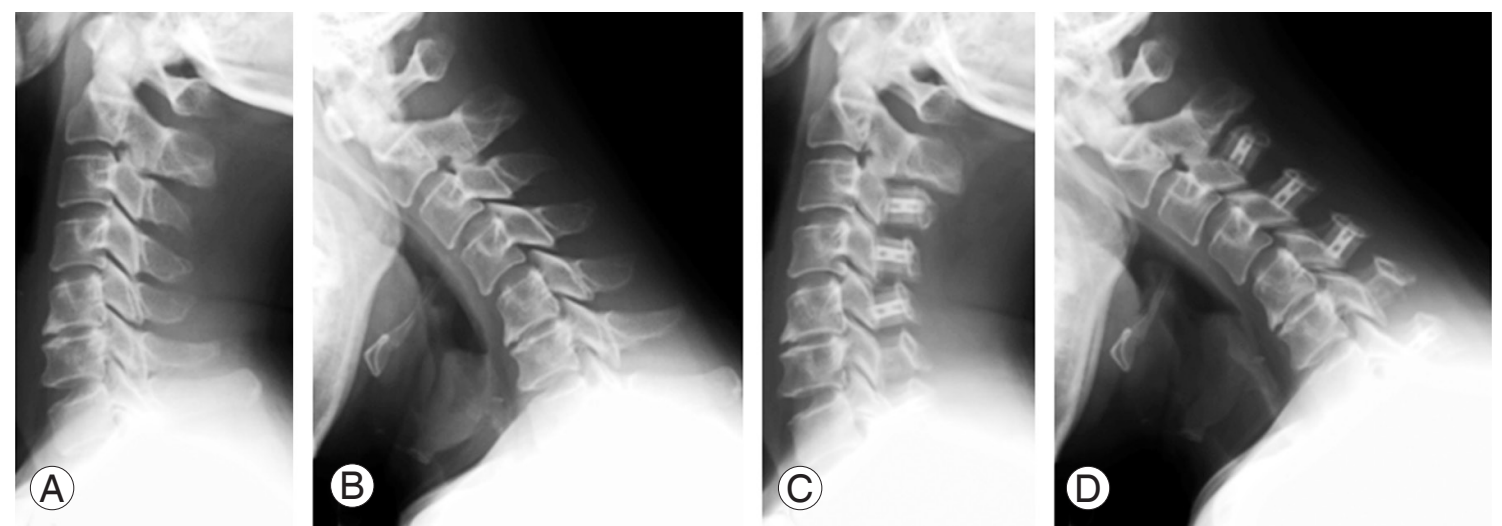

Fig. 7. Pre- (A, B) and postoperative (C, D) flexion-extension views of the cervical spine. The preoperative ROM of the cervical spine was $58^{\circ}$, and the postoperative ROM was $53^{\circ} ; 90 \%$ of the preoperative ROM was preserved. ROM, range of motion

\section{Discussion}

Expansive cervical laminoplasty is an optimal operative procedure for correcting developmental spinal canal stenosis, spinal cord compression at multiple levels, or multi-level disc disease of the cervical spine. This process enables sufficient decompression of the cervical spine while preserving the mobility of the cervical spine, but consistent reconstruction of the expanded laminae of the vertebral arches is still warranted to ensure clinical recovery from the symptoms as well as to prevent postoperative kyphosis of the cervical spine. The successful reconstruction of the laminae is required to accomplish these results because the sinking or non-union of the expanded laminae may result in neurological regression, segmental motor paralysis, and the so-called 'axial pain'

Several modified methods of expansive laminoplasty have been developed for the rigid reconstruction of the expanded laminae, involving anchoring screws, plates, and HA spacers [6,15-17]. These tools enable cervical laminoplasty with a short operation time and low morbidity rate while ensuring a short duration of postoperative immobilization of the neck. However, most of these devices do not demonstrate adaptability to the bone tissues. Lee et al. [18] reported hinge fusion failure after plate-only open-door laminoplasty and concluded that the absence of a ventral cortical bony continuity at 6 months is a risk factor for hinge fusion failure even in the long term, that is, at 12 months postoperatively.

IP-CHA was developed as an artificial bone substitute with sufficient biocompatibility and bone-conduction capability $[12,13]$, and it has been clinically used to compensate for bone defects with bone-inductive substances
[9,11]. Despite its excellent biocompatibility and superior osteoconductivity, IP-CHA itself may not have sufficient mechanical strength to hold the expanded laminae. In our previous study, wherein we applied uniform IP-CHA as a spacer for expansive laminoplasty [7], 1.8\% of the grafted IP-CHA spacers showed breakages despite postoperative neck immobilization and deliberate rehabilitation, indicating its fragile nature in the early postoperative days when used alone.

In the present study, hybrid HA spacer was a composite of porous and solid materials, that is, IP-CHA and solid ceramic, and showed both sufficient initial strength and excellent osteoconductivity. A structure without an adhesive layer was realized at the boundary between the porous and solid materials using an innovative integral molding process. The shape of the hybrid HA spacer was designed to suit 'open-door' laminoplasty. These spacers possess an asymmetrical and shallow shape to conform to the 'open side' of the expanded laminae and not interfere with one another spacer during neck movement.

In the present study, we reported the clinical results, including those associated with bonding rates and the spacer-related complications of cervical laminoplasty, obtained using hybrid HA spacers. Satisfactory clinical results with RRs of $52 \%$ were obtained without any complications related to the use of the hybrid HA spacers, and the postoperative ROM of the cervical spine was $80 \%$ preserved without any axial neck pain. Postoperative CT revealed excellent bone bonding of the hybrid HA spacers equivalent to that of an autogenous bone. Moreover, the hybrid HA exhibited no adsorption, whereas $16 \%$ of the autogenous spacers were absorbed within 6 months postoperatively. In addition, the hinges of the laminae ex- 
panded using hybrid HA spacers showed sufficient bone bonding throughout the follow-up period, suggesting that they are adequately strong to hold the expanded laminae. Furthermore, their unique shape, which fits to the expanded laminae, may also contribute to the high fusion rate of the hybrid HA spacers. Moreover, the hybrid HA spacers could supply adequate support for early exercise of the cervical spine, whereas the stable reconstruction of the expanded laminae preserved the postoperative movement of the cervical spine and maintained good sagittal alignment.

Recent technological advances have enabled the development of more favorable products in which different elements are fused; these 'hybrids' are believed to produce synergistic effects. In the present study, spacers comprising both IP-CHA and solid ceramic demonstrated sufficient bone-bonding rate and yielded satisfactory clinical results after cervical laminoplasty without any major complications. The hybridization of the conventional materials may underlie a revolution in therapeutic procedures for spine surgeries in the near future.

However, this study has some limitations. First, it was conducted at a single center with a small sample size. Second, our results may not accurately reflect precise clinical results after cervical laminoplasty using the hybrid HA spacers. In addition, the follow-up period may be insufficient to examine the long-term clinical efficacy of the spacers. Therefore, further prospective studies demonstrating the clinical efficacy of the proposed procedure for patients with cervical myelopathy are needed.

\section{Conclusions}

Hybrid HA spacers comprising IP-CHA and solid ceramic contributed to high bone-fusion rates with the spacers and hinges of the laminae, and no major complications were associated with their use. Although the long-term clinical results are unknown, cervical laminoplasty with hybrid HA spacers can be a safe and simple method to yield sufficient fixation strength and ensure sufficient bone bonding within a short period of time after operation.

\section{Conflict of Interest}

No potential conflict of interest relevant to this article was reported.

\section{References}

1. Hase H, Watanabe T, Hirasawa Y, et al. Bilateral open laminoplasty using ceramic laminas for cervical myelopathy. Spine (Phila Pa 1976) 1991;16:1269-76.

2. Hirabayashi S, Kumano K. Contact of hydroxyapatite spacers with split spinous processes in double-door laminoplasty for cervical myelopathy. J Orthop Sci 1999;4:264-8.

3. Koshu K, Tominaga T, Yoshimoto T. Spinous process-splitting laminoplasty with an extended foraminotomy for cervical myelopathy. Neurosurgery 1995;37:430-4.

4. Nakano K, Harata S, Suetsuna F, Araki T, Itoh J. Spinous process-splitting laminoplasty using hydroxyapatite spinous process spacer. Spine (Phila Pa 1976) 1992;17(3 Suppl):S41-3.

5. Goto T, Ohata K, Takami T, et al. Hydroxyapatite laminar spacers and titanium miniplates in cervical laminoplasty. J Neurosurg 2002;97(3 Suppl):323-9.

6. Park AE, Heller JG. Cervical laminoplasty: use of a novel titanium plate to maintain canal expansion: surgical technique. J Spinal Disord Tech 2004;17:26571.

7. Tanaka N, Nakanishi K, Fujimoto Y, et al. Expansive laminoplasty for cervical myelopathy with interconnected porous calcium hydroxyapatite ceramic spacers: comparison with autogenous bone spacers. J Spinal Disord Tech 2008;21:547-52.

8. Park YK, Lee DY, Hur JW, Moon HJ. Delayed hinge fracture after plate-augmented, cervical open-door laminoplasty and its clinical significance. Spine J 2014;14:1205-13.

9. Adachi N, Ochi M, Deie M, Ito Y. Transplant of mesenchymal stem cells and hydroxyapatite ceramics to treat severe osteochondral damage after septic arthritis of the knee. J Rheumatol 2005;32:1615-8.

10. Ito Y, Tanaka N, Fujimoto Y, et al. Bone formation using novel interconnected porous calcium hydroxyapatite ceramic hybridized with cultured marrow stromal stem cells derived from Green rat. J Biomed Mater Res A 2004;69:454-61.

11. Nakasa T, Ishida O, Sunagawa T, et al. Prefabrication of vascularized bone graft using a combination of fibroblast growth factor- 2 and vascular bundle implantation into a novel interconnected porous calcium hydroxyapatite ceramic. J Biomed Mater Res A 
$2005 ; 75: 350-5$

12. Tamai N, Myoui A, Tomita T, et al. Novel hydroxyapatite ceramics with an interconnective porous structure exhibit superior osteoconduction in vivo. J Biomed Mater Res 2002;59:110-7.

13. Yoshikawa $\mathrm{H}$, Myoui A. Bone tissue engineering with porous hydroxyapatite ceramics. J Artif Organs 2005;8:131-6.

14. Hirabayashi K, Miyakawa J, Satomi K, Maruyama T, Wakano K. Operative results and postoperative progression of ossification among patients with ossification of cervical posterior longitudinal ligament. Spine (Phila Pa 1976) 1981;6:354-64.

15. Wang JM, Roh KJ, Kim DJ, Kim DW. A new method of stabilising the elevated laminae in open-door laminoplasty using an anchor system. J Bone Joint Surg Br 1998;80:1005-8.

16. Lee JY, Hanks SE, Oxner W, Tannoury C, Donaldson WF 3rd, Kang JD. Use of small suture anchors in cervical laminoplasty to maintain canal expansion: a technical note. J Spinal Disord Tech 2007;20:33-5.

17. Satomi K, Ogawa J, Ishii Y, Hirabayashi K. Shortterm complications and long-term results of expansive open-door laminoplasty for cervical stenotic myelopathy. Spine J 2001;1:26-30.

18. Lee S, Chung CK, Kim CH. Risk factor analysis of hinge fusion failure after plate-only open-door laminoplasty. Global Spine J 2015;5:9-16. 